\title{
Air flow characteristics of a room with air vortex diffuser
}

\author{
Marek Borowski ${ }^{1, *}$, Marek Jaszczur ${ }^{2}$, Daniel Satoła ${ }^{2}$, and Michat Karch ${ }^{1}$ \\ ${ }^{1}$ AGH University of Science and Technology, Faculty of Mining and Geoengineering, Kraków, Poland \\ ${ }^{2}$ AGH University of Science and Technology, Faculty of Energy and Fuels, Kraków, Poland \\ ${ }^{3}$ Frapol Sp. z o.o., Kraków, Poland
}

\begin{abstract}
The air diffuser is a very important component of any ventilation system, and the comfort level of ventilated space occupants depend among many other factors on properly designed and choice of diffusers. At present a large number of diffusers are produced to meet a different kind of requirements. One of the most efficient and very popular types of the diffuser is the vortex diffuser in which air flow has angular as well as translational velocity components. This paper investigates experimentally airflow characteristics of vortex ceiling diffuser and its effect on airflow in a ventilated space. Two thermal comfort criteria namely: mean age of the air and ventilation effectiveness have been used to predict the comfort zone inside the room. Effect of supply air velocity on the flow field is investigated and hence the on comfort and energy consumption. The results show that significant amount of energy can be saved by using vortex diffuser in reference to the other diffusers type. The values of the velocity decay coefficient were compared for different diameters of vortex diffuser.
\end{abstract}

\section{Introduction}

The knowledge and forecasts regarding indoor climate conditions are essential for optimising indoor climate and thermal comfort, as well as for energy efficiency and environmental protection [1]. People spend almost $80 \%$ of their time during the day indoors. Therefore, indoor air quality and thermal comfort are two important aspects of the indoor environment that require particular attention.

According ASHARE HVAC [2] design conditions, temperature and relative humidity should be maintained within a range of $20-24^{\circ} \mathrm{C}$ and $40-60 \%$ respectively. Care should also be taken to keep the air clean and control its chemical composition. In addition, indoor air pressure should be maintained, and the air flow in the system is preferred without recirculation [3].

Until now, many researchers have conducted experimental or simulation studies, but not very often simultaneous experimental and simulation studies.

Nielsen [4] described experiments with wall-mounted air supply equipment. In this study, he presented air velocity distributions in the vicinity of a wall. Al-Hamed [5] used computer modelling to simulate room ventilation in order to predict mean air temperatures and air velocities for multiple inlet and outlet locations for air supply to the room. In his considerations, Author took into account the $k-\varepsilon$ turbulence model. The flow field in this model was verified by experimental measurements made in another study .

Srebric and Chen [6] used a simplified method to describe the flow and thermal conditions for eight different types of diffusers: nozzle diffuser, slot (linear) diffuser, valve diffuser, displacement diffuser, round ceiling diffuser, square ceiling diffuser, vortex diffuser, and grille diffuser.

Zhou and Haghighat [7] developed a simplified method for defining boundary conditions at the inlet of a vortex diffuser. They compared airflow visualisation with (Computational Fluid Dynamics) CFD simulations, which showed the effectiveness of the simplified modelling method for a vortex diffuser. They also presented temperature measurement and air velocity profiles in order to validate the results of CFD simulations. This validation showed that the simplified method can be used to model the airflow from diffusers of this type.

On the other hand, Einberga and Hagstrom [8] discussed the results of modelling of multi-cone air diffusers for industrial spaces from CFD. The Authors compared CFD results with experimental measurements. The results of this study showed that the CFD simulation performed with the standard $k-\varepsilon$ model can accurately predict the non-isothermal airflow around the diffuser.

Most of the previous indoor airflow studies were carried out in reduced-size spaces. At the same time, numerical simulations were carried out for the reduced scale in order to compare them with the experiments. Due to the fact that the research study was carried out to a scale, there was a lack of experimental data collected under real conditions in order to validate the mathematical models. From literature survey can be seen that the experimental studies conducted so far were carried out randomly on selected diffuser models.

\footnotetext{
Corresponding author: borowski@agh.edu.pl
} 
The present study focus on the complex geometry of the diffuser in which three-dimensional (3D) flow occurs $[9,10]$. The experimental measurements were performed for 4 diffuser sizes and were made for the air streams flowing through the diffuser from 100 up to $650 \mathrm{~m}^{3} / \mathrm{h}$. The distribution of air velocity in the zone under the diffuser was determined. The study of air diffuser properties and its influence on the indoor airflow distribution was carried out with the use of two approaches: experimental measurements and numerical simulations.

\section{Experimental studies}

\subsection{Experimental set-up}

The general layout of the experimental configuration is presented in Figure 1. The measurement is performed in the room, with an artificial ceiling and with the dimensions of $A \times B=6 \times 6 \mathrm{~m}$, in which the diffuser is installed. The diffuser is connected to a ventilation duct, through which the fan supplies air in the required amount - from $100 \mathrm{~m}^{3} / \mathrm{h}$ to $650 \mathrm{~m}^{3} / \mathrm{h}$. Modification in the air flow rate is carried out using the fan with a speed regulator (inverter). The ceiling is installed in a room with the following dimensions: $\operatorname{axbxc}=7 \times 7 \times 4 \mathrm{~m}$. The studies are carried out on the real scale. Figure 2 presents a photograph of the diffuser installed in the false ceiling.

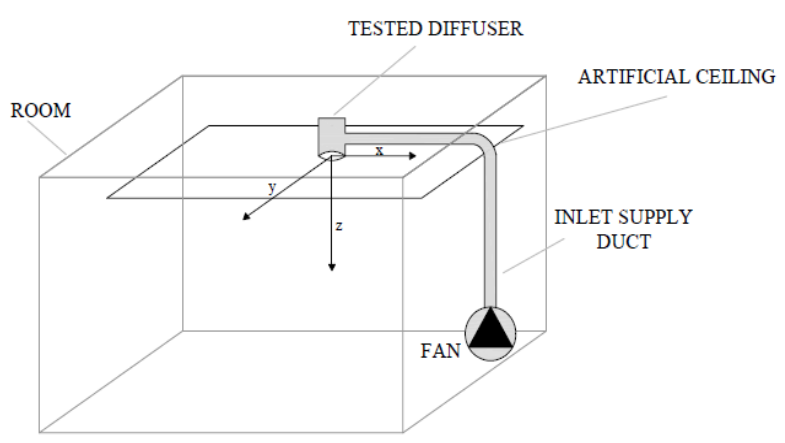

Fig. 1. Schematic diagram of the experimental set-up.

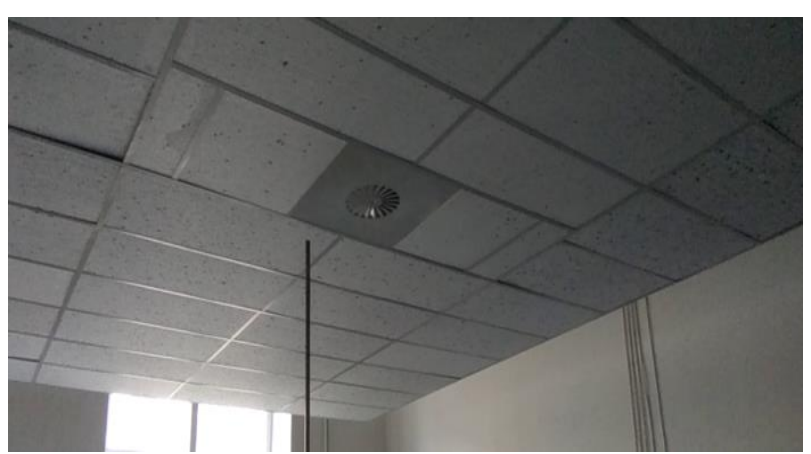

Fig. 2. Diffuser installed in the false ceiling.

The velocity and temperature experimental measurements were made using the AirDistSys 5000 system. This system is used for multi-point measurements of air velocity and temperature. It is used, for example, to assess the thermal conditions and velocity distribution in rooms.

Due to the application of the measuring system of eight SensoAnemo 5100LSF with SF3 probes, measure speed and temperature simultaneously at 8 points with a high level of accuracy. An anemometric transducer measures the airflow velocity in the range from $\mathrm{V}_{\mathrm{w}}=0.05$ to $5.00 \mathrm{~m} / \mathrm{s}$. The technical data of the SensoAnemo 5100LSF are as follows:

- velocity sensor type: multidirectional, spherical,

- speed measurement range: $0.05 \ldots 5.00 \mathrm{~m} / \mathrm{s}$,

- measurement accuracy: $\pm 0.02 \mathrm{~m} / \mathrm{s} \pm 1.5 \%$,

- directional sensitivity error above $2 \mathrm{~m} / \mathrm{s}: \pm 2.5 \%$,

- temperature measurement range: $-10 \ldots+50^{\circ} \mathrm{C}$,

- measurement accuracy: $\pm 2^{\circ} \mathrm{C}$.

The system also includes the AirDistSys 5000 computer software, which enables the configuration of equipment, measurement of mean air velocity and temperature, the standard deviation of velocity, the intensity of flow turbulence and the degree of draught risk at all analysed measurement points. With the acquisition system, all parameters are recorded and stored on a computer. The data can be recorded at any time and for any length of time.

The measurements were made on a grid of points in the $\mathrm{X}-\mathrm{Z}$ plane, as shown in Figure 3.

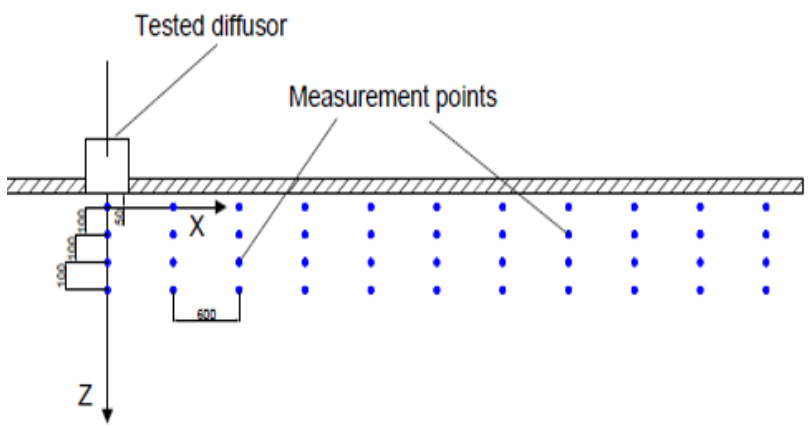

Fig. 3. Measurement points in the X-Z plane.

The measurements were taken in the so-called "lines", i.e. first the measurements were taken on one line for all air streams, and then the thermoanemometers were lowered to the next line along the Z-axis. In the case of the studied diffuser, the first measurement was made at a distance $\mathrm{Z}=0.05 \mathrm{~m}$ from the false ceiling. Then, the distance from the ceiling was gradually increased by $0.1 \mathrm{~m}$. The next measurement lines were at the distance of $Z=0.15 ; 0.25 ; 0.35 \mathrm{~m}$.

\subsection{Methodology}

A ceiling diffuser, which is available in various sizes, was used for the measurements. The geometry of the diffuser is presented in Figure 4.

Each diffuser was installed in a false ceiling and measured for different air streams. The range of the inlet air velocity of the diffuser ranged from $\mathrm{V}_{\mathrm{m}}=0.5$ to $4 \mathrm{~m} / \mathrm{s}$. The test was carried out as follows: after starting the fan and setting the required air flow through the diffuser, the measurement waited until the statistically steady 
conditions were obtained. Commencing the measurements depends on the degree of temperature stabilisation. The condition is considered steady when the temperature is the same within $2 \%$ between two consecutive readings of the measurements. The stationary state is reached after approx. 15 minutes. Then the measurements of air velocity along the determined measurement lines using thermoanemometric probes are started. The duration of the measurement, in which the mean air velocity result was obtained, amounted to 3 minutes. After these measurements, further measurements are taken for the subsequent air streams/air velocities flowing through the diffuser. Then the entire measurement procedure is repeated from the beginning.

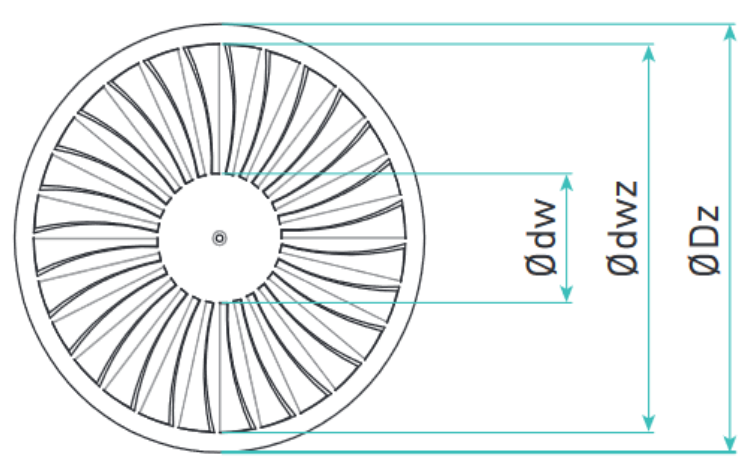

Fig. 4. Simulated diffusers geometry.

The diffusers used in experimental analysis are made in four sizes: 300, 400, 500, $600 \mathrm{~mm}$. Each of them has different dimensions, which are presented in Table 1.

Table 1. List of the characteristic dimensions of diffusers.

\begin{tabular}{|c|c|c|c|}
\hline \multirow{2}{*}{ Size } & $\boldsymbol{d} \boldsymbol{w}$ & $\boldsymbol{d} \boldsymbol{w z}$ & $\boldsymbol{D} \boldsymbol{z}$ \\
\cline { 2 - 4 } & $\mathrm{mm}$ & $\mathrm{mm}$ & $\mathrm{mm}$ \\
\hline 300 & 85 & 250 & 300 \\
\hline 400 & 115 & 350 & 400 \\
\hline 500 & 150 & 450 & 500 \\
\hline 600 & 180 & 550 & 600 \\
\hline
\end{tabular}

\section{Results and discussion}

The measurements for the size 300 diffuser were made for 6 air flow rates $\mathrm{V}=100,150,200,250,300$ and 350 $\mathrm{m}^{3} / \mathrm{h}$. The measurements were made at eight points with thermoanemometers suspended on a horizontal crossbar from $X=0.0 \mathrm{~m}$ to $\mathrm{X}=2.7 \mathrm{~m}$. The measurement points (thermoanemometers) were located at a distance of 0.3 $\mathrm{m}$ from each other. The first thermoanemometer was located on the axis of the diffuser $X=0.00 \mathrm{~m} ; \mathrm{Z}=0.05 \mathrm{~m}$.

Figure 5 shows an example of the velocity distribution for the air flow rates equal to $\mathrm{V}=250 \mathrm{~m}^{3} / \mathrm{h}$. It can be infer from the figure that the largest changes in the air flow occur at the distance from the false ceiling in the plane $\mathrm{Z}=0.05 \mathrm{~m}$. At a larger greater distance from the ceiling, the air velocity is smaller than $0.2 \mathrm{~m} / \mathrm{s}$. The analysis shows that the air stream range for the speed of $0.2 \mathrm{~m} / \mathrm{s}$ equals $\mathrm{L}_{0.2}=2.5 \mathrm{~m}$. It should be noted that the diffuser has a significant air stream range.

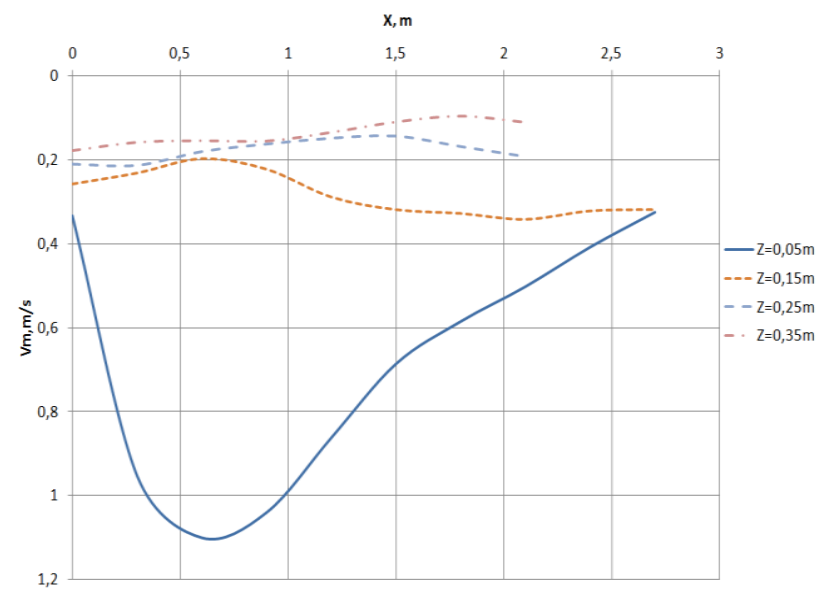

Fig. 5. Velocity distribution for a size 300 diffuser for the air flow rate $\mathrm{V}=250 \mathrm{~m}^{3} / \mathrm{h}$.

Due to the fact that the most significant changes take place on the plane $Z=0.05 \mathrm{~m}$, Figure 6 shows the summary breakdown of the velocity distributions for different air flow rates. This figure shows that the maximum velocity in this plane occurs at a horizontal distance of about $0.75 \mathrm{~m}$ and then decreases to disappear at a distance of $1.4 \mathrm{~m}$ to $3.5 \mathrm{~m}$, depending on the actual air flow rate.

Figure 7 presents the air velocity profile of the air flowing out of the size 300 diffuser in the plane of $\mathrm{Z}=0.05 \mathrm{~m}$ from the ceiling and for the flow rate equal to $\mathrm{V}=100 \mathrm{~m}^{3} / \mathrm{h}$. This figure shows the characteristic shape of the velocity profile from the diffuser.

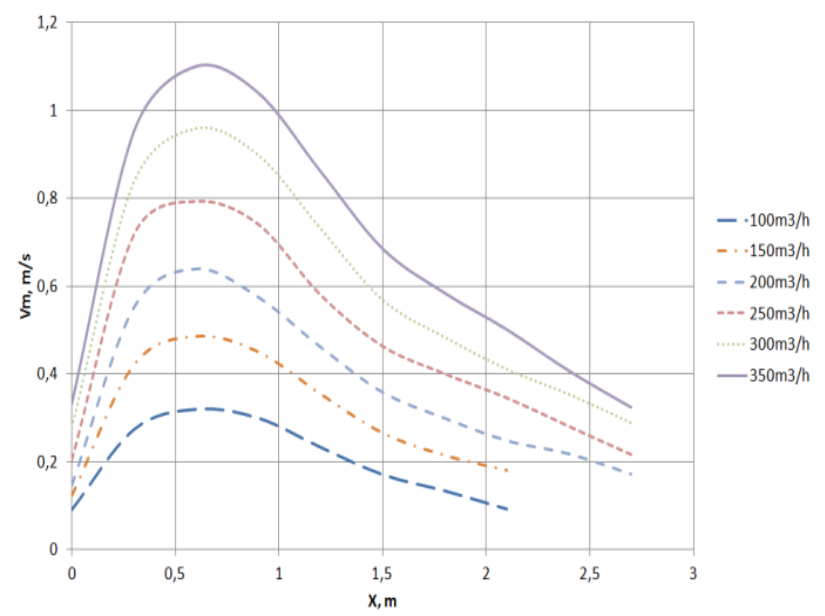

Fig. 6. Velocity distribution for a size 300 diffuser for different air flow rates and in the plane $Z=0.05 \mathrm{~m}$. 

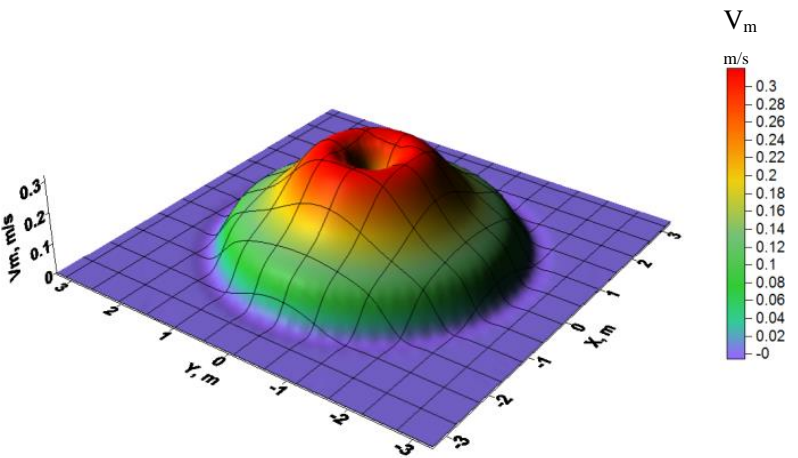

Fig. 7. Velocity profile for a size 300 diffuser in the plane $\mathrm{Z}=0.05 \mathrm{~m}$ for air flow rate $\mathrm{V}=100 \mathrm{~m}^{3} / \mathrm{h}$.

\subsection{Description of the air flow from the ceiling diffuser}

Figure 8 presents the distribution of velocity from the vortex diffuser with the blades at an angle of $45^{\circ}$. The swirl of the air stream from this diffuser causes the air stream to spread under the ceiling. It seems that the vertical range of the diffuser is low. However, the horizontal range is significant with such a small stream of air and dimensions of the diffuser. This is a signal of the high efficiency of air distribution in the room. One can also observe the swirling motion of the air in the room.

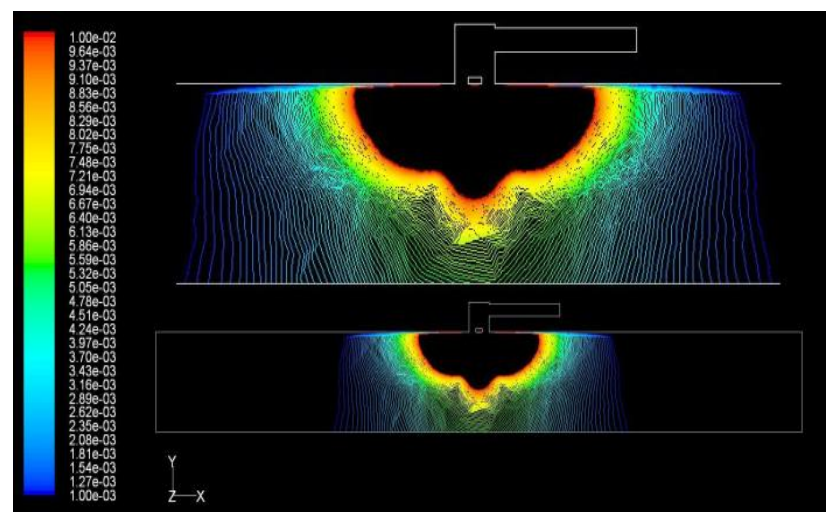

Fig. 8. Vortex diffuser air velocity iso-lines for $\mathrm{V}_{\mathrm{m}}=0.65 \mathrm{~m} / \mathrm{s}$.

\subsection{Diffuser velocity decay coefficient}

The performance of the jet created by the diffuser can be characterized by a single constant, namely jet velocity decay coefficient $K$. It can be defined as [11]:

$$
\frac{V}{V_{o}}=K \frac{\sqrt{A_{o}}}{x}
$$

The diffuser velocity decay coefficient $K$ can be determined by plotted the dimensionless velocity $\mathrm{V} / \mathrm{V}_{\mathrm{o}}$ versus the dimensionless distance $x /$ sqrt $\left(A_{o}\right)$ as shown in Figure 9 where the values of the decay coefficient can be directly determined when the curve intersects the dimensionless velocity at the unity (i.e. $\mathrm{V} / \mathrm{V}_{\mathrm{o}}=1$ ). The value of the velocity decay coefficient is 1.6 .

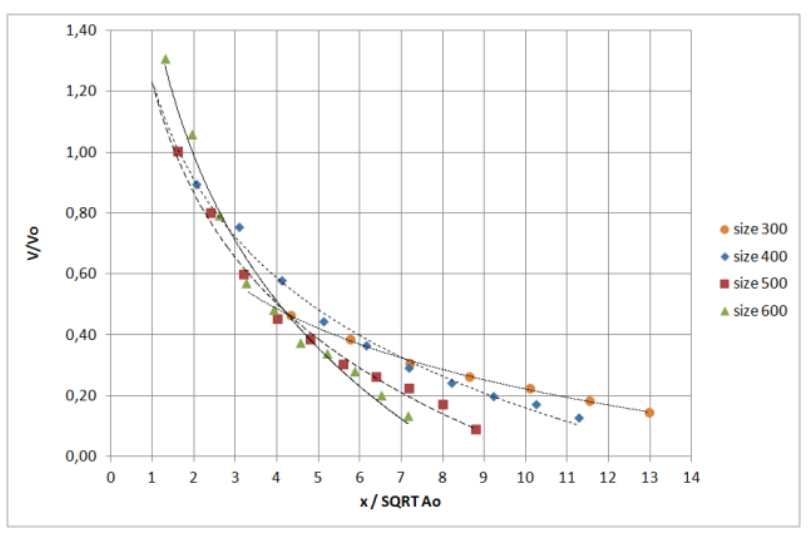

Fig. 9. Dimensionless velocity for different size for vortex diffuser

\section{Conclusion}

This article presents the results of experimental and numerical studies for different air flow rates and thus different inlet velocities of air into a vortex diffuser.

The velocity vector of the studied vortex diffuser is mainly directed horizontally under the ceiling. The long range is achieved by the swirling movement of the air leaving the diffuser. After the air is distributed under the ceiling, it drops, and the heat gains in the room are observed. In this way, the comfort conditions in the room are effectively ensured. Due to the high horizontal air range, no dead zones are formed in the room. The airflow is also distributed in such a way that the air circulation in the room is reduced. It can be seen that the vortex diffuser is characterised by high efficiency of indoor air distribution.

The buoyancy force has a significant impact on the temperature and velocity field, the diffuser creates a thermal comfort zone with a large radius.

The airflow range for the 300 size vortex diffuser for $0.2 \mathrm{~m} / \mathrm{s}$ is in the range $\mathrm{L}_{0.2}=1.4-3.5 \mathrm{~m}$. The diffuser has a large range of the air stream.

The maximum velocity under the false ceiling is about $0.75 \mathrm{~m}$ horizontally and then decreases to the loss of flow $\mathrm{V}=0.2 \mathrm{~m} / \mathrm{s}$ depending on the air stream value.

The pressure coefficient rises sharply for a Reynolds number of less than $\mathrm{Re}=10,000$. An analysis of the dimensionless mean velocity $\mathrm{V}_{\mathrm{m}} / \mathrm{V}_{\mathrm{o}}$ in relation to the Reynolds Number Re shows that it also rises below 10,000. For small airflows and thus low Reynolds numbers, it is difficult to ensure the correct air stream profile and indoor air distribution and thus the right conditions for thermal comfort. 


\section{References}

1. Awbi H. B, Ventilation of Buildings, second ed., (Spon Press. Publisher: Taylor \& Francis 2003)

2. ASHRAE Handbook, Fundamentals, (2008)

3. ASHRAE Standards, Standard 55, (1992)

4. P. V. Nielsen, Energy and Buildings 31, 179 (2002)

5. Al-Hamed, A. H. Determining Velocity, Temperature and Occupancy Comfort within a 3-D. ROOM, (M.Sc. Thesis, King Fahd University, Saudi Arabia 1990)

6. J. Srebric, Q. Chen, HVAC\&R Research, 8, 277 (2002)

7. Zhou L, Haghighat F, Civil and Environmental Engineering, Concordia University, Montreal, Canada, (2007)

8. Einberga G, Hagstrom $\mathrm{K}$, Building and Environment, 40, 601 (2005)

9. M. Jaszczur, M. Branny, M. Karch, M. Borowski, Journal of Physics. Conference Series; ISSN 17426588, 745, 032049, 1 (2016)

10. M. Jaszczur, M. Borowski, M. Branny, M. Karch, The study of the velocity field of the air flowing the swirl diffusers using PIV method, Experimental Fluid Mechanics 2016: proceedings of the international conference, Mariánskè Lázně, Czech Republic, Technical University of Liberec, (2016)

11. Mierzwiński S, Aerodynamics for ventilation, (in Polish, Publisher: Pol. Śląskiej. Gliwice 2007) 